\title{
Spectrum of renal biopsies; a three-year data from a tertiary care centre of eastern India
}

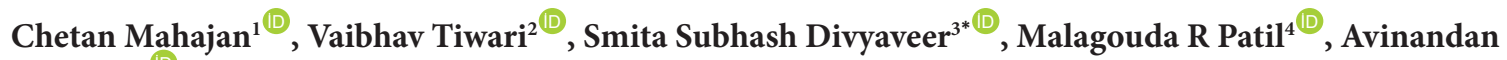 \\ Banerjee $^{1} \mathbb{E}$, Vishnuvardhan Bagur ${ }^{1}$, Umesh Dubey ${ }^{1}$, Koushik Battacharjee ${ }^{1}$, Sandeep Saini ${ }^{1}$, Tanima Das \\ Bhattacharya $^{1 \mathbb{D}}$, Arpita Raychaudhury ${ }^{\mathbb{D}}$, Sanjay Dasgupta ${ }^{1}$, Dipankar Sarkar ${ }^{\mathbb{D}}$, Anila Abraham \\ Kurien $^{5}$, Rajendra Pandey ${ }^{1}$
}

${ }^{1}$ Institute of Post-Graduate Medical Education and Research, Kolkata, India

${ }^{2}$ Sir Ganga Ram Hospital, Institute of Post-Graduate Medical Education and Research, Delhi, India

${ }^{3}$ Post-Graduate Medical Institute of Education and Research, Chandigarh, India

${ }^{4}$ Karnataka Institute of Medical Sciences, Hubli, India

${ }^{5}$ Centre for Renal and Urological Pathology, Chennai, Tamil Nadu, India

\section{A R T I C L E I N F O}

Article Type:

Original

\section{Article History:}

Received: 17 September 2019

Accepted: 23 December 2019

Published online: 20 January 2020

Keywords:

Renal biopsy

AKI

Chronic kidney disease

Nephrotic syndrome

Glomerulonephritis

Thrombotic microangiopathy

End-stage renal disease

\begin{abstract}
A B S T R A C T
Introduction: Biopsy registries are important in studying the frequency of kidney diseases, their trends over time and allow comparison of data from different geographical regions.

Objectives: We report the spectrum of biopsy-proven glomerular and tubular diseases in a single centre in Eastern India.

Patients and Methods: Medical records of 1293 patients with biopsy-proven glomerular diseases (GDs) and tubular diseases over a period of three years (March 2013 to March 2016) were retrospectively analysed. All biopsy specimens were examined by the same pathologist with light and immunofluorescence microscopy. Electron microscopic (EM) analysis was performed only in selected cases. Histologic spectrum of various GDs was studied along with its correlation with the clinical and laboratory parameters.

Results: The clinical diagnosis was nephrotic syndrome (NS) in 820 (63.41\%), rapidly progressive glomerulonephritis in 194 (15.2\%), asymptomatic urinary abnormalities (AUA) in 108 (8\%), acute kidney injury (AKI)/acute nephritic syndrome in 118 (9.3\%), and macroscopic hematuria (MH) in 3 $(0.43 \%)$ patients. Male: Female ratio was 0.95 . Around $22.42 \%$ were $<18$ years, $72.2 \%$ were between 18 to 59 years, and $5.3 \%$ were $\geq 60$ years of age. The most common GD overall as well as primary glomerular disease (PGD) was minimal change disease (MCD) (21.6 and 83.44\%). Secondary GD was present in 20.87\%; most common being lupus nephritis (84.58\%). Among the NS, the most common GD was MCD (33.17\%), followed by FSGS (17.56\%), membranous nephropathy (MN) (13.90\%), lupus nephritis (LN) (9.39\%), IgA-nephropathy (0.49\%), and MPGN (7.32\%). Thrombotic microangiopathy (TMA) constituted $24.6 \%$ of AKI presentation particularly common in pregnancy. Diffuse proliferative glomerulonephritis (DPGN) with severe renal dysfunction was present in $14 \%$ of elderly patients.

Conclusion: The spectrum of GD varies according to the area of study and changes over time. Some entities which have not been reported earlier from this region that are uncommon but are significant were pregnancy associated TMA, C3 glomerulopathy (C3G) and DPGN in adults.
\end{abstract}

\section{Implication for health policy/practice/research/medical education:}

This study presents the pattern of kidney diseases based on biopsy findings. It will help in analyzing the changes in kidney diseases, especially glomerular diseases over time and comparing different disease patterns in other developing and developed countries. Understanding clinical and biopsy correlations will help in management of patients. Preventive and treatment strategies can further be planned for newly recognized disease entities.

Please cite this paper as: Mahajan C, Tiwari V, Divyaveer SS, Patil MR, Banerjee A, Bagur V, et al. Spectrum of renal biopsies; a three-year data from a tertiary care centre of eastern India. J Nephropharmacol. 2020;9(2):e20. DOI: 10.15171/npj.2020.20.

\section{Introduction}

Renal biopsy registries provide the data on epidemiology of various renal pathologies and provide insight into the changing trends over time $(1,2)$. These data also allow comparison of pattern of renal diseases between different geographical regions across the world (1). In Japan the incidence of diabetic nephropathy is rising, while IgA nephropathy is showing a declining trend (3). The biopsy registries help in estimating the disease burden due to individual diseases and this may help primary and 
secondary prevention strategies. In India, population based data on chronic kidney disease (CKD) is not very robust however, a high overall prevalence of $17.2 \%$ has been reported (4). Among the causes of CKD, diabetic nephropathy (31\%) has been reported as the commonest followed by CKD of unknown origin (16\%) (5). Screening programs for urinary abnormalities and conducting the biopsy in early stages will help define the glomerular diseases (GDs) in the CKD with unknown etiology $(\mathrm{CKDu})$ group. The developments in the understanding of pathogenesis of various kidney diseases resulted in changes and updating of classification systems applied to the reporting of renal biopsies (6,7). Additionally, some newer entities like C3 glomerulopathy have been defined (8). Despite an encouraging data on non-invasive biomarkers, renal biopsy is an irreplaceable investigation, essential for prognosis and treatment in many diseases at present (9). Though invasive, in the experience of most centers it has been found safe with very low risk of complication in both adults and children $(10,11)$. Hence, renal biopsy registries are an essential tool for epidemiologic analysis as well as for enhancing and continuing our understanding of the differences in renal diseases across various regions and ethnicities (12).

\section{Objectives}

There is data on spectrum of renal biopsies from various regions of India, however, from eastern India is scarce $(13,14)$. Here, we present the observations and analysis of three years data of renal biopsies performed at the largest single center of Eastern India. The aim was to study the spectrum of renal biopsies and the corresponding clinical presentation. This study also elaborates our protocol of renal biopsy which may be of paramount importance in identifying GDs at early stage and thereby unraveling the $\mathrm{CKDu}$ group further.

\section{Patients and Methods \\ Study design}

This is a retrospective observational single center study of the native kidney biopsies performed between March 2013 to March $2016(\mathrm{n}=1293)$ at Institute of Post-Graduate Medical Education and Research, Kolkata. Records of renal biopsies performed during this period were examined. For collection of data regarding the clinical and laboratory parameters of renal biopsy $(\mathrm{Bx})$ requisitions containing age, gender and indication of biopsy were reviewed. The data obtained was analyzed and biopsy findings were compared with clinical and laboratory parameters.

The analysis of biopsy registry and its comparisons with other studies, heavily depends on the biopsy practices of the institute/geographical area. Hence we consider it prudent to detail the protocol of renal biopsy indications practiced at our institute. The indications of renal biopsy in childhood onset nephrotic syndrome adopted in our institute are the guidelines recommended by Kidney
Disease Improving Global Outcomes (KDIGO) (15). Additional indications in childhood onset nephrotic syndrome as well as in other clinical presentations have been elaborated in Table S1 (See online Supplementary file 1). Acute kidney injury (AKI) in pregnancy which is not associated with preeclampsia or other obvious cause is biopsied unless contraindicated to diagnose hemolytic uremic syndrome. There is no population based urinary screening program in this part of the country however, those incidentally detected to have asymptomatic urinary abnormalities (AUA) suggestive of glomerular etiology are conducted kidney biopsy as this would help in prognostication and treatment of the disease. The contraindications to biopsy are mentioned in Table S2.

\section{Renal biopsy procedure}

As an institutional protocol, two cores of renal tissue were obtained with automated biopsy gun $(16 \mathrm{G} \times 16 \mathrm{~cm}$ for adults $\geq 18$ years old and $18 \mathrm{G} \times 16 \mathrm{~cm}$ for patients $<18$ years of age) under real-time ultrasonography guidance and subjected to light microscopy (LM) using Hematoxylin and eosin, periodic acid-Schiff (PAS) stain, methenamine silver stain and trichrome stain and immunofluorescence (IF).

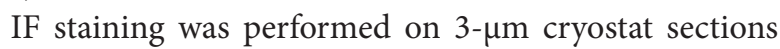
using polyclonal fluorescein isothiocyanate-conjugated antibodies to IgG, IgM, IgA, C3, C1q, kappa and lambda light chains. The intensity of IF staining was graded on a scale of 0 to $3+$. Interstitial fibrosis and tubular atrophy (IFTA) were graded as mild $(<25 \%)$, moderate $(25-50 \%)$, and severe $(>50 \%)$ on LM. When thought necessary by the treating physician electron microscopy was done using the paraffin embedded tissue. Additional special stains were used whenever indicated. All biopsy samples were analyzed by a single nephropathologist. A second biopsy was performed in case a biopsy sample was inadequate for diagnosis and if exact diagnosis would have significant implications on the therapy and if the patients gave consent for a re-biopsy.

\section{Ethical considerations}

This is a retrospective study. The existing records of data available with department of nephrology, IPGMER, Kolkata were reviewed and analyzed. The research followed the tenets of the Declaration of Helsinki. Written informed consent at the time of renal biopsy was obtained.

\section{Statistical analysis}

Descriptive statistical analysis was performed. For categorical data, proportion is presented as percentage of total. Continuous normally distributed data are presented as means with corresponding standard deviations (SD).

Results

We first describe the clinical presentation of patients undergoing kidney biopsy and the baseline characteristics 
followed by the etiological diagnosis in each clinical presentation category. For further analysis of our biopsy data, kidney diseases have been classified as; glomerular pathologies including; (a) primary glomerular diseases (PGD) and (b) secondary glomerular diseases (SGD). Patients with evidence of extensive chronic glomerulosclerosis (CGS) were also classified into PGD or SGD depending on the findings on histology. If they were not classifiable as PGD or SGD, they have been included separately as CGS. Finally, we compare the data of this study with that of our previous data (16) and the data from neighbouring regions of our country (16-18) as well as other registries across the globe $(19,20)$.

A total of 1293 biopsy-proven glomerular/tubular diseases were recorded over the period of study. The average glomerular yield was adequate (17.41 \pm 8.42 glomeruli). Electron microscopy (EM) was performed in $5.3 \%$ of biopsies. The average age of the patients was 29.7 \pm 15.24 years. Male: Female ratio was 0.95 (631 males: 662 females).

The contribution of the various glomerular/tubular syndromes to the performance of kidney biopsy is presented in Figure 1. The demographic and baseline characteristics and frequency of each of these syndromes are described in Table 1 . The most common clinical presentation was nephrotic syndrome (NS). The various types of histology and their proportion in each clinical syndrome are presented in Table 2. The most common cause of NS was minimal change disease (MCD) followed by focal segmental glomerulosclerosis (FSGS). The most common etiology of RPRF/RPGN (rapidly progressive renal failure/rapidly progressive glomerulonephritis) was necrotising crescentic GN (CresGN) seen in 32.99\% ( $\mathrm{n}=$ 64); of them, $23.43 \%$ were anti-GBM disease and $18.75 \%$ were pauci-immune. The next common GD presenting as RPRF/ RPGN was lupus nephritis (LN) $(32.47 \%, \mathrm{n}=63)$, of which class IV was in $71.42 \%$ of cases. Lupus nephritis was seen in $9.39 \%(n=77)$ of NS while $55.84 \%$ of the cases were either pure class V of LN or class V overlap with other LN classes. The distribution of various etiologies to GN with

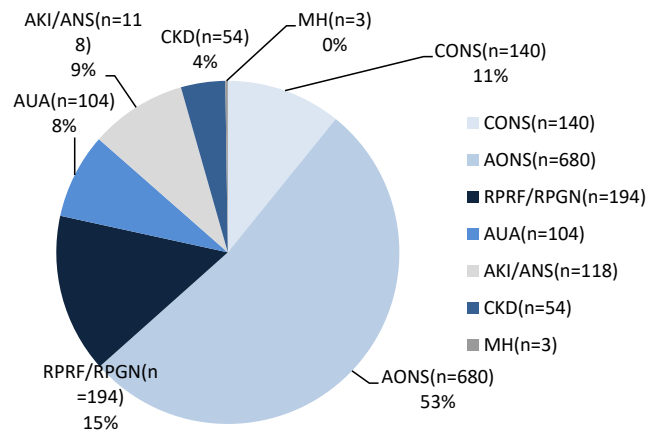

Figure 1. Spectrum of various clinical syndromes.

crescents has been shown in Table S3. As expected, the disease with the severest changes was necrotising CresGN, with an average of $79.03 \pm 33.2 \%$ crescents on biopsy. Four cases of MN showed crescents. Of these two had full house staining on immunofluorescence (IF) suggestive of secondary MN but has no clinical or serological evidence of a secondary disease. The variants of FSGS have been shown in Table S4. Membranoproliferative GN (MPGN) comprised $7.35 \%$ of the total biopsies.

In those presenting as AKI/AGN, acute tubular necrosis/acute tubulointerstitial nephritis (ATN/ATIN) and thrombotic microangiopathy (TMA) contributed to $24.58 \%$ each followed by lupus nephritis. In ATN/ ATIN group $72.41 \%(n=21)$ cases were none recovering AKI associated with snake bite. One case was diarrhoea associated non-recovering AKI. Rest of the cases were of undetermined etiology. Two cases had moderate chronicity (IFTA $>50 \%$ ). In our study $75.8 \%$ cases of TMA were presented as post-partum AKI. Around 17.2\% of cases were associated with malignant hypertension. About $68.18 \%$ of patients in post-partum TMA had patchy cortical necrosis. Of, all TMA cases, one case had calcineurin inhibitor toxicity and one had post-diarrhoeal haemolytic uremic syndrome. The most common cause of AUA was IgAN contributing to almost half (42\%) of the cases. IgAN was also the most common etiological

Table 1. Baseline characteristics of patients in each clinical syndrome $(n=1293)$

\begin{tabular}{|c|c|c|c|c|c|c|c|}
\hline Variable & $\begin{array}{c}\text { CONS } \\
(n=140)\end{array}$ & $\begin{array}{c}\text { AONS } \\
(n=680)\end{array}$ & $\begin{array}{c}\text { RPRF/RPGN } \\
(n=194)\end{array}$ & $\begin{array}{l}\text { AKI/ANS } \\
(n=118)\end{array}$ & $\begin{array}{c}\text { AUA } \\
(n=104)\end{array}$ & $\begin{array}{c}\text { CKD } \\
(n=54)\end{array}$ & $\begin{array}{c}\text { MH } \\
(n=3)\end{array}$ \\
\hline Age $(y)^{*}$ & $10.5 \pm 5.0$ & $31.8 \pm 14.4$ & $33.3 \pm 15.1$ & $31.0 \pm 14.9$ & $29.9 \pm 12.0$ & $36 \pm 16.0$ & $25.3 \pm 12.5$ \\
\hline Gender (male) & 61.4 & 50.7 & 36.6 & 38.1 & 49.5 & 51.8 & 66.6 \\
\hline Hypertension (\% in each category) & 0.07 & 41.3 & 77.8 & 75.6 & 31.8 & 90.8 & 0 \\
\hline Serum creatinine* $(\mathrm{mg} / \mathrm{dL})$ & $0.9 \pm 0.42$ & $1.64 \pm 1.49$ & $5.2 \pm 4.3$ & $5.1 \pm 3.2$ & $1.6 \pm 0.9$ & $6.67 \pm 4.14$ & $1.13 \pm 0.2$ \\
\hline Serum albumin* (g/dL) & $2.7 \pm 0.6$ & $2.5 \pm 0.9$ & $2.8 \pm 1.1$ & $2.9 \pm 1.4$ & $3.1 \pm 0.8$ & $2.8 \pm 0.7$ & $2.9 \pm 0.5$ \\
\hline Hemoglobin* (g/dL) & $12.4 \pm 2.8$ & $10.9 \pm 3.6$ & $8.8 \pm 2.7$ & $11.1 \pm 2.2$ & $11.5 \pm 1.5$ & $7.9 \pm 2.9$ & $12.7 \pm 0.8$ \\
\hline Hematuria (\% in each category) & 14.2 & 26.3 & 82.1 & 70.6 & 66.1 & 18.5 & 100 \\
\hline 24 hour Urine Protein* $(\mathrm{g} / \mathrm{d})$ & $3.5 \pm 2.12$ & $4.7 \pm 3.0$ & $2.7 \pm 1.8$ & $1.2 \pm 0.9$ & $1.7 \pm 1.1$ & $2.4 \pm 1.3$ & $0.6 \pm 0.4$ \\
\hline
\end{tabular}

*Values indicate mean \pm standard deviation

RPRF, Rapidly progressive renal failure; RPGN, Rapidly progressive glomerulonephritis; AUA, Asymptomatic urinary abnormalities; ANS, Acute nephritic syndrome; AKI, Acute kidney injury; CKD, Chronic kidney disease; $\mathrm{MH}$, Macroscopic hematuria. 
Table 2. Spectrum of various glomerular histologies in each clinical syndrome

\begin{tabular}{|c|c|c|c|c|c|c|}
\hline $\begin{array}{l}\text { Biopsy Diagosis } \\
\mathrm{n}(\% \text { of total) }\end{array}$ & $\begin{array}{c}\text { NS }(n=820) \\
(\%)\end{array}$ & $\begin{array}{l}\text { RPRF/RPGN } \\
(n=194)(\%)\end{array}$ & $\begin{array}{c}\text { AKI/ANS } \\
(n=118)(\%)\end{array}$ & $\begin{array}{c}\text { AUA } \\
(n=104)(\%)\end{array}$ & $\begin{array}{c}\text { CKD } \\
(n=54)(\%)\end{array}$ & $\begin{array}{c}\text { MH } \\
(n=3)(\%)\end{array}$ \\
\hline MCD/Normal ( $n=279 ; 21.58 \%)$ & $272(33.2)$ & $0(0)$ & $5(4.2)$ & $2(1.9)$ & $0(0)$ & $0(0)$ \\
\hline FSGS ( $n=163 ; 12.61 \%)$ & $144(17.6)$ & $3(1.5)$ & $6(5.1)$ & $8(7.7)$ & $2(3.7)$ & $0(0)$ \\
\hline $\mathrm{MN}(\mathrm{n}=132 ; 10.21 \%)$ & $114(13.9)$ & $7(3.6)$ & $3(2.5)$ & $6(5.8)$ & $2(3.7)$ & $0(0)$ \\
\hline LN ( $n=181 ; 14.00 \%)$ & $77(9.4)$ & $63(32.5)$ & $19(16.1)$ & $21(20.2)$ & $1(1.9)$ & $0(0)$ \\
\hline $\operatorname{IgAN} / \mathrm{HSP}(\mathrm{n}=134 ; 10.36 \%)$ & $59(7.2)$ & $10(5.2)$ & $3(2.5)$ & $42(40.4)$ & $17(31.5)$ & $3(100)$ \\
\hline MPGN (n=95; 7.35\%) & $60(7.3)$ & $14(7.2)$ & $9(7.6)$ & $5(4.8)$ & $7(13.0)$ & $0(0)$ \\
\hline DPGN (n=98; 7.58\%) & 49 (5.9) & $32(16.5)$ & $5(4.2)$ & $11(10.6)$ & $1(1.9)$ & $0(0)$ \\
\hline CresGN (n=79;6.11 \%) & $4(0.5)$ & $64(33.0)$ & $7(5.9)$ & $0(0)$ & $4(7.4)$ & $0(0)$ \\
\hline $\operatorname{PCD}(n=29 ; 2.24 \%)$ & $23(2.8)$ & $0(0)$ & $1(0.8)$ & $0(0)$ & $5(9.3)$ & $0(0)$ \\
\hline CGS (n=19; $1.47 \%)$ & $5(0.6)$ & $0(0)$ & $0(0)$ & $0(0)$ & $14(25.9)$ & $0(0)$ \\
\hline NGS $(n=4 ; 0.31 \%)$ & $4(0.5)$ & $0(0)$ & $0(0)$ & $0(0)$ & $0(0)$ & $0(0)$ \\
\hline MesPGN ( $n=16 ; 1.24 \%)$ & $8(1.0)$ & $0(0)$ & $2(1.7)$ & $6(5.8)$ & $0(0)$ & $0(0)$ \\
\hline TMA $(n=29 ; 2.24 \%)$ & $0(0)$ & $0(0)$ & $29(24.6)$ & $0(0)$ & $0(0)$ & $0(0)$ \\
\hline ATN/ATIN (n=30; 2.32\%) & $0(0)$ & $0(0)$ & $29(24.6)$ & $1(1.0)$ & $0(0)$ & $0(0)$ \\
\hline C1qN (n=1;0.08\%) & $1(0.1)$ & $0(0)$ & $0(0)$ & $0(0)$ & $0(0)$ & $0(0)$ \\
\hline Lithium toxicity ( $n=1 ; 0.08 \%)$ & $0(0)$ & $0(0)$ & $0(0)$ & $0(0)$ & $1(1.9)$ & $0(0)$ \\
\hline DN ( $n=1 ; 0.08 \%)$ & $0(0)$ & $0(0)$ & $0(0)$ & 1 & $0(0)$ & $0(0)$ \\
\hline Collapsing glomerulopathy ( $n=1 ; 0.08 \%)$ & $0(0)$ & $1(0.5)$ & $0(0)$ & $0(0)$ & $0(0)$ & $0(0)$ \\
\hline Hypertensive nephrosclerosis ( $n=1 ; 0.08 \%$ ) & $0(0)$ & $0(0)$ & $0(0)$ & $1(1.0)$ & $0(0)$ & $0(0)$ \\
\hline
\end{tabular}

NS, Nephrotic syndrome; RPRF, Rapidly progressive renal failure; RPGN, Rapidly progressive glomerulonephritis; AUA, Asymptomatic urinary abnormalities; ANS, Acute nephritic syndrome; AKI, Acute kidney injury; CKD, Chronic kidney disease; MH, Macroscopic hematuria; MCD, Minimal change disease; FSGS, Focal segmental glomerulosclerosis; MN, Membranous nephropathy; IgAN, IgA nephropathy; HSP, Henoch-Schönlein purpura; MPGN, Membranoproliferative glomerulonephritis; DPGN, Diffuse proliferative glomerulonephritis; CresGN, Crescentic glomerulonephritis; FPGN, Focal proliferative glomerulonephritis; PCD, Plasma Cell Dyscracia; CGS, Chronic glomerulosclerosis; NGS, Nodular glomerulosclerosis; LCDD, Light chain deposition disease; TMA, Thrombotic microangiopathy; DN, Diabetic nephropathy; C1qN, C1q nephropathy; MesPGN, Mesangial proliferative glomerulonephritis; TBMD, Thin basement membrane disease; LN, Lupus nephritis; ATIN, Acute tubulointerstitial nephritis.

diagnosis (17\%) in those presenting as CKD. In 14\% cases of CKD no definitive diagnosis was possible. These patients have been classified as CGS. IgAN/ HenochSchönlein purpura was the diagnosis in all case of macroscopic hematuria $(\mathrm{MH})$.

In diffuse proliferative glomerulonephritis (DPGN), the majority of the cases were adults especially $>60$ years. The most common presentation was NS (49.5\%) although a significant number (32.9\%) presented with RPRF with dialysis requirement. A small number of patients also presented as infection related glomerulonephritis with incomplete recovery of renal function. The different biopsy diagnosis in the monoclonal gammopathy group as a common disease in adults especially elderly (including plasma cell dyscrasias) has been shown in Table S5. We had also a case of idiopathic nodular glomerulosclerosis.

Glomerular diseases were also classified according to gender and age at presentation ( $<12$ years, $12-17$ years, 18 59 years, and $\geq 60$ years) (Tables 3 and 4 respectively). The GDs were also studied based on serum creatinine values (Table 5). Serum creatinine $\geq 3 \mathrm{mg} / \mathrm{dL}$ was common in CresGN, IgAN and DPGN. MCD was the most common GD in the age groups of $<12$ and $12-17$ years, whereas LN was the most common GD in adults in the age group 1859 years and $\mathrm{MN}$ was the commonest GD in the elderly patients.

The distribution of GD as PGD and SGD is presented in Table 6 and Figure 2. LN was the most common SGD. MCD was the most common GD followed by FSGS and IgAN.

\section{Discussion}

Analysis of CKD trends, suggests that despite diabetes and hypertension being the most common causes of endstage renal disease (ESRD), incidence of GDs contributing to ESRD is also rising (21). Biopsy registries serve many purposes as elaborated in introduction above.

The most frequent indication for performing renal biopsy in our study was similar to most single centre reports from across the world was NS (22-24) While single centre studies almost invariably have NS as the most common indication $(25,26)$. This may largely be due to urinary screening strategies adopted by these nations. However, in Japan where population based urinary screening programs have been implemented, since chronic glomerulonephritis was the most common indication there (27). AUA was the 
Table 3. Glomerular disease spectrum according to gender

\begin{tabular}{|c|c|c|c|c|}
\hline Biopsy Diagnosis & Male $(n=631)$ & female $(n=662)$ & $\begin{array}{c}\text { Sex ratio } \\
\text { F:M }\end{array}$ & $\begin{array}{c}\text { Sex ratio } \\
\text { M:F }\end{array}$ \\
\hline MCD/Normal & $161(25.5 \%)$ & $118(17.8 \%)$ & - & $1.36: 1$ \\
\hline FSGS & 112 (17.7\%) & $51(7.7 \%)$ & - & $2.19: 1$ \\
\hline $\mathrm{MN}$ & 78 (12.36\%) & $54(8.2 \%)$ & - & $1.4: 1$ \\
\hline LN & $20(3.1 \%)$ & $161(24.3 \%)$ & $8.05: 1$ & - \\
\hline IgAN/HSP & $74(11.7 \%)$ & 60 (9.1\%) & - & $1.23: 1$ \\
\hline MPGN & $43(6.81 \%)$ & $52(7.8 \%)$ & $1.20: 1$ & - \\
\hline DPGN & $46(7.3 \%)$ & $52(7.8 \%)$ & $1.13: 1$ & - \\
\hline CresGN & $32(5.1 \%)$ & 47 ( (7.1\%) & $1.46: 1$ & - \\
\hline PCD & $17(2.7 \%)$ & $12(1.8 \%)$ & - & $1.41: 1$ \\
\hline CGS & $8(1.2 \%)$ & $11(1.6 \%)$ & $1.3: 1$ & - \\
\hline NGS & $3(0.5 \%)$ & $1(0.2 \%)$ & - & $3: 1$ \\
\hline MesPGN & $8(1.2 \%)$ & $8(1.2 \%)$ & - & $1: 1$ \\
\hline TMA & $7(1.1 \%)$ & $22(3.3 \%)$ & $3.14: 1$ & - \\
\hline ATN/ATIN & $18(2.9 \%)$ & $12(1.8 \%)$ & - & $1.5: 1$ \\
\hline $\mathrm{C} 1 \mathrm{qN}$ & $1(0.2 \%)$ & 0 & - & - \\
\hline Lithium toxicity & & $1(0.2 \%)$ & - & - \\
\hline $\mathrm{DN}$ & $1(0.2 \%)$ & 0 & - & - \\
\hline Collapsing glomerulopathy & $1(0.2 \%)$ & 0 & - & - \\
\hline Hypertensive nephrosclerosis & $1(0.2 \%)$ & 0 & - & - \\
\hline
\end{tabular}

MCD, minimal change disease; FSGS, focal segmental glomerulosclerosis; MN, membranous nephropathy; IgAN, IgA nephropathy; HSP, HenochSchönlein purpura; MPGN, membranoproliferative glomerulonephritis; DPGN, diffuse proliferative glomerulonephritis; CresGN, crescentic glomerulonephritis; PCD, plasma cell dyscracia; CGS, chronic glomerulosclerosis; NGS, nodular glomerulosclerosis; TMA, thrombotic microangiopathy; DN, diabetic nephropathy; C1qN, C1q nephropathy; MesPGN, mesangial proliferative glomerulonephritis; LN, lupus nephritis; ATIN, acute tubulointerstitial nephritis.

Table 4. Glomerular disease spectrum according to the age of presentation

\begin{tabular}{|c|c|c|c|c|c|c|c|c|}
\hline \multirow{3}{*}{$\begin{array}{l}\text { Biopsy Diagnosis } \\
\text { MCD/normal }\end{array}$} & \multicolumn{8}{|c|}{ Age Group (y) } \\
\hline & \multicolumn{2}{|c|}{$<12$} & \multicolumn{2}{|c|}{$12-17$} & \multicolumn{2}{|c|}{$18-59$} & \multicolumn{2}{|c|}{$>60$} \\
\hline & 78 & $69.0 \%$ & 65 & $36.7 \%$ & 133 & $14.2 \%$ & 3 & $4.3 \%$ \\
\hline FSGS & 10 & $8.8 \%$ & 31 & $17.5 \%$ & 115 & $12.3 \%$ & 7 & $10.1 \%$ \\
\hline $\mathrm{MN}$ & 4 & $3.5 \%$ & 8 & $4.5 \%$ & 107 & $11.5 \%$ & 13 & $18.8 \%$ \\
\hline LN & 2 & $1.8 \%$ & 19 & $10.7 \%$ & 159 & $17.0 \%$ & 1 & $1.4 \%$ \\
\hline IgAN/HSP & 5 & $4.4 \%$ & 9 & $5.1 \%$ & 116 & $12.4 \%$ & 4 & $5.8 \%$ \\
\hline MPGN & 4 & $3.5 \%$ & 14 & $7.9 \%$ & 69 & $7.4 \%$ & 8 & $11.6 \%$ \\
\hline DPGN & 4 & $3.5 \%$ & 10 & $5.6 \%$ & 73 & $7.8 \%$ & 10 & $14.5 \%$ \\
\hline FPGN & 0 & $0.0 \%$ & 0 & $0.0 \%$ & 1 & $0.1 \%$ & 0 & $0.0 \%$ \\
\hline CresGN & 1 & $0.9 \%$ & 9 & $5.1 \%$ & 60 & $6.4 \%$ & 9 & $13.0 \%$ \\
\hline PCD & 0 & $0.0 \%$ & 0 & $0.0 \%$ & 21 & $2.2 \%$ & 8 & $11.6 \%$ \\
\hline CGS & 0 & $0.0 \%$ & 3 & $1.7 \%$ & 16 & $1.7 \%$ & 0 & $0.0 \%$ \\
\hline NGS & 0 & $0.0 \%$ & 0 & $0.0 \%$ & 3 & $0.3 \%$ & 1 & $1.4 \%$ \\
\hline MesPGN & 1 & $0.9 \%$ & 4 & $2.3 \%$ & 11 & $1.2 \%$ & 0 & $0.0 \%$ \\
\hline TMA & 3 & $2.7 \%$ & 1 & $0.6 \%$ & 25 & $2.7 \%$ & 0 & $0.0 \%$ \\
\hline ATN/ATIN & 0 & $0.0 \%$ & 4 & $2.3 \%$ & 22 & $2.4 \%$ & 4 & $5.8 \%$ \\
\hline $\mathrm{C} 1 \mathrm{qN}$ & 1 & $0.9 \%$ & 0 & $0.0 \%$ & 0 & $0.0 \%$ & 0 & $0.0 \%$ \\
\hline Lithium toxicity & 0 & $0.0 \%$ & 0 & $0.0 \%$ & 1 & $0.1 \%$ & 0 & $0.0 \%$ \\
\hline DN & 0 & $0.0 \%$ & 0 & $0.0 \%$ & 1 & $0.1 \%$ & 0 & $0.0 \%$ \\
\hline Collapsing glomerulopathy & 0 & $0.0 \%$ & 0 & $0.0 \%$ & 0 & $0.0 \%$ & 1 & $1.4 \%$ \\
\hline Hypertensive nephrosclerosis & 0 & $0.0 \%$ & 0 & $0.0 \%$ & 1 & $0.1 \%$ & 0 & $0.0 \%$ \\
\hline
\end{tabular}

MCD, minimal change disease; FSGS, focal segmental glomerulosclerosis; MN, membranous nephropathy; IgAN, IgA nephropathy; HSP, HenochSchönlein purpura; MPGN, membranoproliferative glomerulonephritis; DPGN, diffuse proliferative glomerulonephritis; CresGN, crescentic glomerulonephritis; PCD, plasma cell dyscracia; CGS, chronic glomerulosclerosis; NGS, nodular glomerulosclerosis; TMA, thrombotic microangiopathy; DN, diabetic nephropathy; C1qN, C1q nephropathy; MesPGN, mesangial proliferative glomerulonephritis; LN, lupus nephritis; ATIN, acute tubulointerstitial nephritis. 
Table 5. Spectrum of glomerular diseases and distribution in various serum creatinine (SCr) levels

\begin{tabular}{|c|c|c|c|c|c|c|}
\hline \multirow{2}{*}{$\begin{array}{l}\text { Biopsy Diagnosis } \\
\text { MCD/Normal }\end{array}$} & \multicolumn{2}{|c|}{$\mathrm{SCr}<1.5 \mathrm{mg} / \mathrm{dL}$} & \multicolumn{2}{|c|}{$\mathrm{SCr} 1.5-2.9 \mathrm{mg} / \mathrm{dL}$} & \multicolumn{2}{|c|}{$\mathrm{SCr}>3.0 \mathrm{mg} / \mathrm{dL}$} \\
\hline & 245 & $34.5 \%$ & 28 & $8.8 \%$ & 6 & $2.3 \%$ \\
\hline FSGS & 90 & $12.7 \%$ & 68 & $21.3 \%$ & 5 & $1.9 \%$ \\
\hline MN & 107 & $15.0 \%$ & 19 & $6.0 \%$ & 6 & $2.3 \%$ \\
\hline LN & 93 & $13.1 \%$ & 64 & $20.1 \%$ & 24 & $9.1 \%$ \\
\hline IgAN/HSP & 58 & $8.2 \%$ & 43 & $13.5 \%$ & 33 & $12.5 \%$ \\
\hline MPGN & 51 & $7.2 \%$ & 22 & $6.9 \%$ & 22 & $8.4 \%$ \\
\hline DPGN & 36 & $4.9 \%$ & 34 & $10.7 \%$ & 28 & $10.6 \%$ \\
\hline CresGN & 3 & $0.4 \%$ & 12 & $3.8 \%$ & 64 & $24.3 \%$ \\
\hline PCD & 16 & $2.3 \%$ & 5 & $1.6 \%$ & 8 & $3.0 \%$ \\
\hline CGS & 0 & $0.0 \%$ & 2 & $0.6 \%$ & 17 & $6.5 \%$ \\
\hline NGS & 1 & $0.1 \%$ & 2 & $0.6 \%$ & 1 & $0.4 \%$ \\
\hline MesPGN & 9 & $1.3 \%$ & 5 & $1.6 \%$ & 2 & $0.8 \%$ \\
\hline TMA & 0 & $0.0 \%$ & 3 & $0.9 \%$ & 26 & $9.9 \%$ \\
\hline ATN/ATIN & 1 & $0.1 \%$ & 10 & $3.1 \%$ & 19 & $7.2 \%$ \\
\hline $\mathrm{C} 1 \mathrm{qN}$ & 1 & $0.1 \%$ & 0 & $0.0 \%$ & & $0.0 \%$ \\
\hline Lithium toxicity & 0 & $0.0 \%$ & 0 & $0.0 \%$ & 1 & $0.4 \%$ \\
\hline DN & 0 & $0.0 \%$ & 1 & $0.3 \%$ & 0 & $0.0 \%$ \\
\hline Collapsing glomerulopathy & 0 & $0.0 \%$ & 1 & $0.3 \%$ & 0 & $0.0 \%$ \\
\hline Hypertensive Nephrosclerosis & 0 & $0.0 \%$ & 0 & $0.0 \%$ & 1 & $0.4 \%$ \\
\hline
\end{tabular}

MCD, minimal change disease; FSGS, focal segmental glomerulosclerosis; MN, membranous nephropathy; IgAN, IgA nephropathy; HSP, HenochSchönlein purpura; MPGN, membranoproliferative glomerulonephritis; DPGN, diffuse proliferative glomerulonephritis; CresGN, crescentic glomerulonephritis; PCD, plasma cell dyscracia; CGS, chronic glomerulosclerosis; NGS, nodular glomerulosclerosis; TMA, thrombotic microangiopathy; DN, diabetic nephropathy; C1qN, C1q nephropathy; MesPGN, mesangial proliferative glomerulonephritis; LN, lupus nephritis; ATIN, acute tubulointerstitial nephritis.

most common indication of biopsy in studies from Italy and France (28).

According to hygiene hypothesis, the increased exposure to bacterial antigens in developing countries may reflect a different pattern of response of the immune system, which translates in a different exacerbation of particular GDs [higher prevalence of MPGN, in developing areas, higher prevalence of IgA-nephropathy (IgAN), and MCD, in more developed countries $(26,29)$. India has a growing burden of problems of either extremes of economical classes and hence it is not possible to extrapolate the data from other countries to India directly $(30,31)$. Prevalence of MPGN in our data was lower than that reported from developing countries like Romania (32). As cited above in India $\mathrm{CKDu}$ as a significant prevalence and most if not all are likely to be due to GDs that went undetected in early stages. As is shown in Table 7, our centre has reported higher prevalence of IgAN compared to our own earlier data as well as other regions of India. This may be a reflection of rather more vigorous biopsy practice in our institute in an effort to define the etiology of GD even when the clinical presentation is that of AUA or CKD. In both of these clinical syndromes and those with $\mathrm{MH}$, IgAN was the most frequent diagnosis. Biopsy in CKD

PRIMARY $\square$ SECONDARY

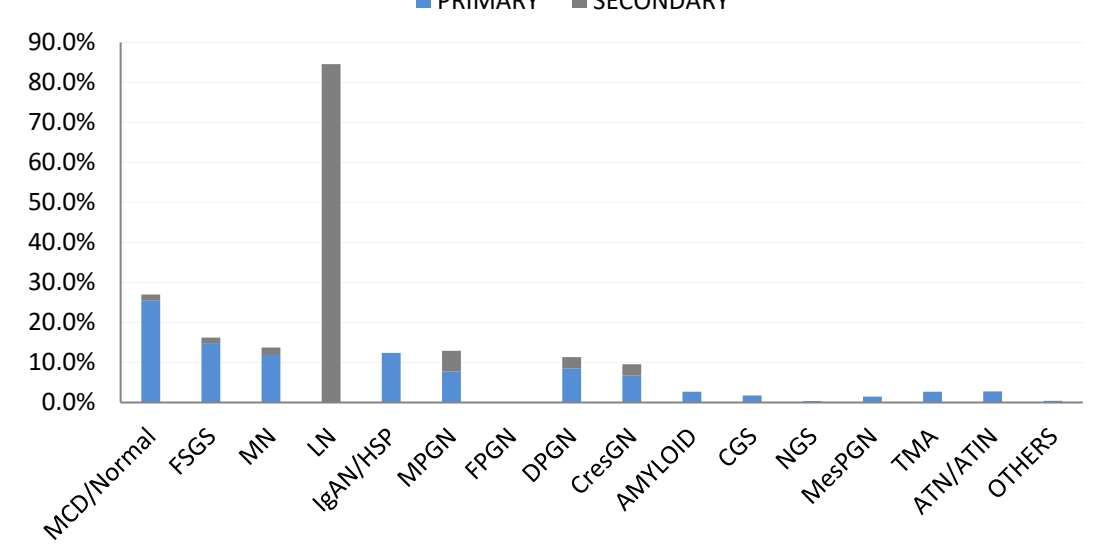

Figure 2. Primary and secondary glomerular diseases. 
Table 6. Frequencies of primary and secondary glomerular diseases

\begin{tabular}{lccc}
\hline Biopsy diagnosis & Total & $\begin{array}{c}\text { Primary } \\
\mathbf{n}=\mathbf{1 0 7 9}(\%)\end{array}$ & $\begin{array}{c}\text { Secondary } \\
\mathbf{n}=\mathbf{2 1 4}(\%)\end{array}$ \\
\hline MCD/Normal & 279 & $276(25.58)$ & $3(1.40)$ \\
FSGS & 163 & $160(14.83)$ & $3(1.40)$ \\
MN & 132 & $128(11.86)$ & $4(1.80)$ \\
LN & 181 & $0(0)$ & $181(84.58)$ \\
IgAN/HSP & 134 & $134(12.42)$ & $0(0)$ \\
MPGN & 95 & $84(7.78)$ & $11(5.14)$ \\
DPGN & 98 & $92(8.53)$ & $6(2.80)$ \\
CresGN & 79 & $73(6.76)$ & $6(2.80)$ \\
PCD & 29 & $29(2.69)$ & $0(0)$ \\
CGS & 19 & $19(1.76)$ & $0(0)$ \\
NGS & 4 & $4(0.37)$ & $0(0)$ \\
MesPGN & 16 & $16(1.48)$ & $0(0)$ \\
TMA & 29 & $29(2.69)$ & $0(0)$ \\
ATN/ATIN & 30 & $30(2.78)$ & $0(0)$ \\
C1qN & 1 & $1(0.09)$ & $0(0)$ \\
Lithium toxicity & 1 & $1(0.09)$ & $0(0)$ \\
DN & 1 & $1(0.09)$ & $0(0)$ \\
\hline Collapsing Glomerulopathy & 1 & $1(0.09)$ & $0(0)$ \\
Hypertensive nephrosclerosis & 1 & $1(0.09)$ & $0(0)$ \\
\hline & & & \\
\hline
\end{tabular}

is considered to be controversial (26) but in our study definitive diagnosis was not possible in only $14 \%$ of cases while in the rest of the cases (86\%) a definite etiology was found. The implications of a definitive diagnosis go beyond therapeutics to prognostication of recurrence after transplant if the progresses to ESRD. A population based urinary screening for school children has been recently completed in our institute (unpublished data as yet). This may be the first step in identifying the GN early in their course in our region.

The frequent histological patterns related to primary NS in adults in our study are similar to other studies like MCD, FSGS and MN. However, a significant proportion of IgAN was also presented as NS similar to our previous report and another report from south India but unlike some reports from other countries $(16,18,26,33)$. We did not find an increase in prevalence of FSGS compared to our previous report $(16,23)$, however the gap in the two studies of our institute is too small to define the trends of GD in eastern India. Other studies from India and other countries have reported an increasing prevalence of FSGS $(4,19)$. FSGS of not otherwise specified (NOS) was the most common variant as earlier. The prevalence

Table 7. Comparison of our study with biopsy-proven glomerular disease spectrum seen in other centers in India and neighboring countries

\begin{tabular}{|c|c|c|c|c|c|c|}
\hline Variables & Our Study & $\begin{array}{l}\text { IPGMER } \\
\text { Kolkata }^{16}\end{array}$ & $\begin{array}{c}\text { TITS, } \\
\text { Asarwa }^{23}\end{array}$ & $\begin{array}{l}\text { Karachi, } \\
\text { Pakistan }^{24}\end{array}$ & $\begin{array}{l}\text { RAMAIAH, } \\
\text { Bangalore }^{22}\end{array}$ & $\begin{array}{l}\text { PGIMS, } \\
\text { Rohtak }^{21}\end{array}$ \\
\hline Duration & $2013-2015$ & $2010-2012$ & $2008-2013$ & $2003-2012$ & $2008-2013$ & 2012 \\
\hline No. of biopsies & 1293 & 666 & 335 & 435 & 661 & 75 \\
\hline Age (y) & $29.7 \pm 15.24$ & $28 \pm 14.62$ & $7.91 \pm 3.04$ & 40 & 42.8 & - \\
\hline PGD & 83.45 & 79.13 & 81.79 & - & 42.3 & 61.3 \\
\hline SGD & 16.55 & 20.83 & 16.12 & - & - & 16 \\
\hline MCD/normal & 21.58 & 20.12 & 6.93 & 5 & 14.52 & 8.6 \\
\hline FSGS & 12.61 & 18.02 & 8.02 & 8 & 5.45 & 6.8 \\
\hline $\mathrm{MN}$ & 10.21 & 12.01 & 3.28 & 12 & 6.81 & 25.8 \\
\hline LN & 14.00 & 15.32 & 7.76 & 6 & 3.18 & 12.1 \\
\hline IgAN/HSP & 10.36 & 8.1 & 6.2 & 1.8 & 2.72 & 5.2 \\
\hline MPGN & 7.35 & 5.25 & 13.5 & 8 & 4.08 & 17.2 \\
\hline DPGN & 7.50 & 4.95 & 9.55 & 1.5 & 3.03 & \\
\hline CresGN & 6.11 & 7.51 & 10.94 & 4 & 3.18 & 8.6 \\
\hline PCD & 2.24 & 1.2 & 0.89 & 4 & 0.30 & 6.8 \\
\hline CGS & 1.47 & 3 & - & - & 3.48 & \\
\hline NGS & 0.31 & 0.45 & - & - & 0.00 & - \\
\hline MesPGN & 1.24 & 0.6 & 24.45 & - & 0.00 & - \\
\hline TMA & 2.24 & 1.5 & 6.26 & - & 0.30 & - \\
\hline ATN/ATIN & 2.32 & - & - & 19.5 & 0.15 & - \\
\hline $\mathrm{C} 1 \mathrm{qN}$ & 0.08 & 0.15 & - & - & 0.00 & - \\
\hline Lithium toxicity & 0.08 & - & - & - & 0.00 & - \\
\hline DN & 0.08 & - & - & 7 & 16.64 & 1 \\
\hline Collapsing glomerulopathy & 0.08 & - & - & - & 0.00 & - \\
\hline Hypertensive Nephrosclerosis & 0.08 & - & 1.19 & - & - & - \\
\hline
\end{tabular}

PGD, primary glomerular diseases; SGD, secondary glomerular diseases; MCD, minimal change disease; FSGS, focal segmental glomerulosclerosis; MN, membranous nephropathy; IgAN, IgA nephropathy; HSP, Henoch-Schönlein purpura; MPGN, membranoproliferative glomerulonephritis; DPGN, diffuse proliferative glomerulonephritis; CresGN, crescentic glomerulonephritis; PCD, plasma cell dyscracia; CGS, chronic glomerulosclerosis; NGS, nodular glomerulosclerosis; TMA, thrombotic microangiopathy; DN, diabetic nephropathy; C1qN, C1q nephropathy; MesPGN, mesangial proliferative glomerulonephritis; LN, lupus nephritis; ATIN, acute tubulointerstitial nephritis.

Values indicate $\%$ of total biopsies. 
Table 8. Comparison of our study with biopsy-proven glomerular disease spectrum seen in other centers across the globe

\begin{tabular}{|c|c|c|c|c|}
\hline Variables & Our Study & IPGMER Kolkata ${ }^{16}$ & TITS, Asarwa ${ }^{23}$ & Karachi, Pakistan ${ }^{24}$ \\
\hline Duration & $2013-2015$ & $1994-2011$ & 2010 & $1994-2003$ \\
\hline No. of biopsies & 1293 & 10,472 & 3,869 & 104 \\
\hline Age (y) & $29.7 \pm 15.24$ & 46.6 & $41.3 \pm 15.4$ & \\
\hline PGD (\%) & 83.45 & 57.8 & 55.2 & \\
\hline SGD (\%) & 16.55 & 29 & & \\
\hline MCD/Normal (\%) & 21.58 & 6.1 & & 1.92 \\
\hline FSGS (\%) & 12.61 & 6.9 & 5.1 & 20.1 \\
\hline MN (\%) & 10.21 & 7.1 & 10.2 & 10.5 \\
\hline LN (\%) & 14.00 & 7.1 & 5.4 & 7.69 \\
\hline IgAN/HSP (\%) & 10.36 & 20.5 & 30.4 & 25 \\
\hline MPGN (\%) & 7.35 & 3.2 & 2.4 & 5.76 \\
\hline DPGN (\%) & 7.50 & 0.8 & 0.8 & 7.69 \\
\hline CresGN (\%) & 6.11 & 6.9 & 6.4 & \\
\hline PCD (\%) & 2.24 & 3.7 & 1.4 & \\
\hline CGS (\%) & 1.47 & - & & \\
\hline NGS (\%) & 0.31 & - & & \\
\hline MesPGN (\%) & 1.24 & 3.3 & & \\
\hline TMA (\%) & 2.24 & - & 0.2 & \\
\hline ATN/ATIN (\%) & 2.32 & 3.3 & & \\
\hline C1qN (\%) & 0.08 & - & & \\
\hline Lithium toxicity (\%) & 0.08 & - & & \\
\hline DN (\%) & 0.08 & 4.1 & 4.7 & \\
\hline Collapsing glomerulopathy (\%) & 0.08 & & & \\
\hline Hypertensive nephrosclerosis (\%) & 0.08 & & 3.8 & \\
\hline
\end{tabular}

PGD, primary glomerular diseases; SGD, secondary glomerular diseases; MCD, minimal change disease; FSGS, focal segmental glomerulosclerosis; MN, membranous nephropathy; IgAN, IgA nephropathy; HSP, Henoch-Schönlein purpura; MPGN, membranoproliferative glomerulonephritis; DPGN, diffuse proliferative glomerulonephritis; CresGN, crescentic glomerulonephritis; PCD, plasma cell dyscracia; CGS, chronic glomerulosclerosis; NGS, nodular glomerulosclerosis; TMA, thrombotic microangiopathy; DN, diabetic nephropathy; C1qN, C1q nephropathy; MesPGN, mesangial proliferative glomerulonephritis; LN, lupus nephritis; ATIN, acute tubulointerstitial nephritis.

*Please refer to the details in the actual article given in reference as the categories may be overlapping and the actual \% may hence differ.

of mesangioproliferative GN (MesPGN) is much lower in our data $(1.24 \%)$ compared to that in the other regional studies from India $(18,21,34)$. It has been almost double in number compared to previous study from our institute (16). Unlike our previous report where no case was given the diagnosis as $\mathrm{C} 3$ glomerulopathy (C3G), 5 cases in this study had a diagnosis consistent with $\mathrm{C} 3 \mathrm{G}$, which some of the MesGN cases were C3G.

According to available data, AKI has been associated with increased prevalence of bleeding complications (35) and this may be the reason of hesitation in performing renal biopsy in such settings yet renal biopsy in AKI has varied diagnosis and in about a third of the cases the clinical presentation may not correlate with the diagnosis (36). This is particularly true in case post-partum AKI. While other studies from our country and other neighboring countries (as shown in Tables 7 and 8) have not reported a significant prevalence of TMA except in children we have found this entity in $29 \%$ of all AKI cases. This difference may be due to referral bias where pregnant patient with hospital delivery may be recognized early, referred to nephrologist and readily biopsied. Awareness amongst primary care physicians and obstetricians regarding this disease is still not satisfactory and majority patients are referred late (unpublished data of our institute). This highlights the importance of early renal biopsy in the usually devastating post-partum AKI for early diagnosis, treatment and to decide regarding the prospects of live renal transplant. Also, renal biopsy in AKI can diagnose otherwise covert plasma cell dyscrasia as in one of our cases. Since plasma cell dyscrasias are considered in the differential diagnosis of elderly patients with renal dysfunction, most are diagnosed without kidney biopsy. This is the most likely reason for low prevalence of cast nephropathy compared to other histological diagnoses in this study. The other etiologies of AKI/ANS were similar to those found by Beaman et al (35).

The most common PGD in most registries is IgAN (37) however in our data the most common PGD was MCD followed by FSGS. As discussed above this may be due to subclinical/covert presentation of IgAN. The prevalence of SGD has not changed and correlated well with other biopsy registries with LN being the most common SGD. Diabetic nephropathy is not biopsied routinely hence it contributed only a small proportion of cases. Other important finding was DPGN in elderly (>60 years). Occasionally this may 
be the only presentation of an occult infection. The overall prevalence of DPGN has also increased almost three fold compared to our previous report. This may be due to increasing trend of biopsy in adults with infection related glomerulonephritis. As the prognosis is guarded in this group renal biopsy may prove helpful in diagnosis (38).

A limitation of the study is that EM was not done in all cases. However, in all cases where a definitive diagnosis could not be reached with LM and IF, EM was considered. Yet, the possibility of a different diagnosis after EM remains. Another limitation is that this is a single centre hospital-based biopsy registry so subclinical presentation of disease in the population as less likely to be reflected entirely in our data. Comparisons with other studies may not reflect the true difference in prevalence due to racial/ genetic/environmental/socioeconomic causes but also those due to the differences in indications for renal biopsy.

\section{Conclusion}

Nephrotic syndrome due to MCD is the dominating biopsy finding in this region. The most common cause of AUA was IgAN. The most common glomerular etiology for CKD was IgAN followed by crescentic GN most probably due to late referral. Vigorous biopsy practices as well as population based urinary screening programs may prove extremely help in identifying the etiology of $\mathrm{CKDu}$ in early stages. Although the prevalence of TMA is low, most of the cases had unsatisfactory recovery, emphasizing the early renal biopsy especially in the setting of pregnancy associated AKI for its recognition. MPGN is not as common as found in some developing countries. As compared to the last report from our institute it is seen that the prevalence of DPGN has increased almost three fold. Prevention of infections in elderly and vaccination may help in controlling DPGN in elderly who often have moderate to severe renal dysfunction and usually have guarded prognosis. The prevalence of SGD correlated well with other biopsy registries.

\section{Limitations of the study}

This investigation was retrospective and was conducted in a single center. Besides, the study was cross-sectional without respect to treatment or follow up to find the final outcome of patients. Thus larger studies with multi-center basis are suggested.

\section{Acknowledgments}

We are thankful to all the post-doctoral trainees of IPGMER Kolkata involved in care of these patients.

\section{Authors' contribution}

CM complied, gathered and reviewed the data. SSD analyzed and wrote the primary manuscript. VD organized and analyzed data. AR and RP gave the original idea and have been mentors. AAK reported the renal biopsies. MP,
$\mathrm{AB}, \mathrm{VB}, \mathrm{UD}, \mathrm{KB}, \mathrm{TDS}, \mathrm{SD}$ and $\mathrm{DS}$ were involved in patient care, procedure of renal biopsies and interpretation of renal biopsies. All authors read, revised, and approved the final manuscript.

\section{Conflicts of interest}

The authors declare that they have no conflict of interest.

\section{Ethical considerations}

Ethical issues (including plagiarism, data fabrication, double publication) have been completely observed by the authors.

\section{Funding/Support}

None.

Supplementary Materials

Supplementary file 1 contains Tables S1-S5.

\section{References}

1. Pesce F, Schena FP. Worldwide distribution of glomerular diseases: the role of renal biopsy registries. Nephrol Dial Transplant. 2010;25:334-6. doi: 10.1093/ndt/gfp620.

2. Swaminathan S, Leung N, Lager DJ, Melton LJ 3rd, Bergstralh EJ, Rohlinger A, et al.Changing incidence of glomerular disease in Olmsted County, Minnesota: a 30year renal biopsy study. Clin J Am Soc Nephrol. 2006;1:4837. doi: 10.2215/CJN.00710805.

3. Imai $\mathrm{E}$, Yamagata $\mathrm{K}$, Iseki $\mathrm{K}$, Iso $\mathrm{H}$, Horio $\mathrm{M}$, Mkino $\mathrm{H}$, et al. Kidney disease screening program in Japan: history, outcome, and perspectives. Clin J Am Soc Nephrol. 2007;2:1360-6. doi: 10.2215/CJN.00980207.

4. Singh AK, Farag YM, Mittal BV, Subramanian KK, Reddy SR, Acharya VN, et al. Epidemiology and risk factors of chronic kidney disease in India-results from the SEEK (Screening and Early Evaluation of Kidney Disease) study. BMC Nephrol. 2013;14:114. doi: 10.1186/1471-2369-14114.

5. Rajapurkar MM, John GT, Kirpalani AL, Abraham G, Agarwal SK, Almeida AF, et al. What do we know about chronic kidney disease in India: first report of the Indian CKD registry. BMC Nephrol. 2012;13:10. doi: 10.1186/14712369-13-10.

6. Working Group of the International IgA Nephropathy Network and the Renal Pathology Society, Cattran DC, Coppo R, Cook HT, Feehally J, Roberts IS, et al. The Oxford classification of IgA nephropathy: rationale, clinicopathological correlations, and classification. Kidney Int. 2009;76:534-45. doi: 10.1038/ki.2009.243.

7. Weening JJ, D'agati VD, Schwartz MM, Seshan SV, Alpers CE, Appel GB, et al. The classification of glomerulonephritis in systemic lupus erythematosus revisited. Kidney Int. 2004;65:521-30. doi: 10.1111/j.1523-1755.2004.00443.x.

8. Fakhouri F, Frémeaux-Bacchi V, Noël LH, Cook HT, Pickering MC. C3 glomerulopathy: a new classification. Nat Rev Nephrol. 2010;6:494-9. doi: 10.1038/nrneph.2010.85.

9. Hogan JJ, Mocanu M, Berns JS. The native kidney biopsy: Update and evidence for best practice. Clin J Am Soc Nephrol. 2016;11:354-62. doi: 10.2215/CJN.05750515. 
10. Bairy M, Beleed K, Webb AT, Bhandari S. Safety of outpatient kidney biopsy: one center's experience with 178 native kidney biopsies. Am J Kidney Dis. 2008;52:631-2. doi: $\quad$ 10.1053/j.ajkd.2008.06.019.

11. Tøndel C, Vikse BE, Bostad L, Svarstad E. Safety and complications of percutaneous kidney biopsies in 715 children and 8573 adults in Norway 1988-2010. Clin J Am Soc Nephrol. 2012;7:1591-7. doi: 10.2215/CJN.02150212.

12. Sim JJ, Batech M, Hever A, Harrison TN, Avelar T, Kanter $\mathrm{MH}$, et al. Distribution of biopsy-proven presumed primary glomerulonephropathies in 2000-2011 among a racially and ethnically diverse US population.Am J Kidney Dis. 2016;68:533-544. doi: 10.1053/j.ajkd.2016.03.416.

13. Golay V, Trivedi M, Abraham A, Roychowdhary A, Pandey $\mathrm{R}$. The spectrum of glomerular diseases in a single center: A clinicopathological correlation. Indian J Nephrol. 2013;23:168-175. doi: 10.4103/0971-4065.111833.

14. Rathi M, Bhagat RL, Mukhopadhyay P, Kohli HS, Jha V, Gupta KL, et al. Changing histologic spectrum of adult nephrotic syndrome over five decades in north India: a single center experience. Indian J Nephrol. 2014;24:86-91. doi: 10.4103/0971-4065.127892.

15. Eckardt KU, Kasiske BL. KDIGO Clinical Practice Guideline for Glomerulonephritis Foreword. Kidney Int Suppl. 2012; 2:140. doi: 10.1038/kisup.2012.10.

16. Singh S, Marwah N, Sen R, Sangwan M, Verma R, Ralli M. Profile of renal biopsies in a tertiary care hospital. Int J Healthc Biomed Res. 2014;2:53-9.

17. Devadass CW, Mysorekar VV, Gireesh MS, Mahesh E, Gurudev KC, Radhika K. Review of renal biopsy database: A single centre South Indian study. Int J Med Res Health Sci. 2014;3:959-66.

18. Kanodia KV, Vanikar AV, Nigam LK, Patel RD, Suthar KS, Gera DN, et al. Pediatric renal biopsies in India: a single-centre experience of six years. Nephrourol Mon. 2015;7:e25473. doi: 10.5812/numonthly.25473.

19. Absar A, Asif N, Khan Q, Kashif W. Experience of percutaneus kidney biopsy from a tertiary care center of Pakistan. Open J Nephrol. 2015;5:61.

20. Maixnerova D, Jancova E, Skibova J, Rysava R, Rychlik I, Viklicky $\mathrm{O}$, et al. Nationwide biopsy survey of renal diseases in the Czech Republic during the years 1994-2011. J Nephrol. 2015;28:39-49. doi: 10.1007/s40620-014-0090-z.

21. Fiorentino M, Bolignano D, Tesar V, Pisano A, Van Biesen W, D’Arrigo G, et al. ERA-EDTA Immunonephrology Working Group. Renal biopsy in 2015 - from epidemiology to evidence-based indications. Am J Nephrol. 2016;43:1-9. doi: $10.1159 / 000444026$.

22. Rychlík I, Jančová E, Tesař V, Kolský A, Lácha J, Stejskal J, et al. The Czech registry of renal biopsies. Occurrence of renal diseases in the years 1994-2000. Nephrol Dial Transplant. 2004;19:3040-9. doi: 10.1093/ndt/gfh521.

23. Reshi AR, Bhat MA, Najar MS, Banday KA, Naik MA, Singh DP, et al. Etiological profile of nephrotic syndrome in Kashmir. Indian J Nephrol. 2008;18:9-12. doi: 10.4103/09714065.41281.
24. Garyal, Kafle RK. Hisopathological spectrum of glomerular disease in Nepal: a seven-year retrospective study. Nepal Med Coll J. 2008;10:126-8.

25. Sugiyama H, Yokoyama H, Sato H, Saito T, Kohda Y, Nishi $\mathrm{S}$, et al. Japan renal biopsy registry and Japan kidney disease registry: committee report for 2009 and 2010. Clin Exp Nephrol. 2013;17:155-73. doi: 10.1007/s10157-012-0746-8.

26. Gesualdo L, Di Palma AM, Morrone LF, Strippoli GF, Schena FP. The Italian experience of the national registry of renal biopsies. Kidney Int. 2004;66:890-4.doi: 10.1111/j.15231755.2004.00831.x.

27. Sugiyama H, Yokoyama H, Sato H, Saito T, Kohda Y, Nishi $\mathrm{S}$, et al. Japan renal biopsy registry and Japan kidney disease registry: committee report for 2009 and 2010. Clin Exp Nephrol. 2013;17:155-73. doi: 10.1007/s10157-012-0746-8.

28. Simon P, Ramee MP, Boulahrouz R, Stanescu C, Charasse C, Ang KS, et al. Epidemiologic data of primary glomerular diseases in western France. Kidney Int. 2004;66:905-8. doi: 10.1111/j.1523-1755.2004.00834.x.

29. Hurtado A, Johnson RJ. Hygiene hypothesis and prevalence of glomerulonephritis. Kidney Int Suppl. 2005;68:S62-7. doi: 10.1111/j.1523-1755.2005.09711.x.

30. Chatterjee P. India sees parallel rise in malnutrition and obesity. Lancet. 2002;360:1948. doi: 10.1016/S01406736(02)11943-X.

31. Kshatriya GK, Acharya SK. Triple burden of obesity, undernutrition, and cardiovascular disease risk among Indian tribes. PloS One. 2016;11:e0147934. doi: 10.1371/ journal.pone.0147934.

32. Covic A, Schiller A, Volovat C, Gluhovschi G, GusbethTatomir P, Petrica L, et al. Epidemiology of renal disease in Romania: a 10 year review of two regional renal biopsy databases. Nephrol Dial Transplant. 2006;21:419-24. doi: 10.1093/ndt/gfi207.

33. Quirós PL, Ceballos M, Remón C, Lozano A, del Castillo R, Aznar E, et al. Study of the biopsied nephrotic syndrome for 20 years in the Cadiz bay area: histological correspondence, renal prognosis and clinical prognostic factors. Nefrologia. 2005;25:147-54.

34. Chandra H, Chandra S. Patterns of Glomerulonephritis in Uttarakhand State 13 Years Single Centre Experience at Teaching Institute. Indian Medical Gazette. 2013;220-223.

35. Corapi KM, Chen JL, Balk EM, Gordon CE. Bleeding complications of native kidney biopsy: a systematic review and meta-analysis. Am J Kidney Dis. 2012;60:62-73. doi: 10.1053/j.ajkd.2012.02.330.

36. Beaman M, Turney JH, Rodger RS, McGonigle RS, Adu D, Michael J. Changing pattern of acute renal failure. QJM. 1987;62:15-23.

37. Pesce F, Schena FP. Worldwide distribution of glomerular diseases: the role of renal biopsy registries. Nephrol Dial Transplant. 2010;25:334-6.

38. Nasr SH, Radhakrishnan J, D D’Agati V. Bacterial infection-related glomerulonephritis in adults. Kidney Int. 2013;83:792-803.

Copyright $\odot 2020$ The Author(s); Published by Published by Society of Diabetic Nephropathy Prevention. This is an open-access article distributed under the terms of the Creative Commons Attribution License (http://creativecommons.org/licenses/by/4.0), which permits unrestricted use, distribution, and reproduction in any medium, provided the original work is properly cited. 